\title{
Avaliação de um Algoritmo de Determinação da Taxa de Codificação baseado na qualidade do sinal de voz em uma rede IP emulada
}

\author{
Demóstenes Zegarra Rodríguez e Miguel Arjona Ramírez
}

\begin{abstract}
Resumo-Este trabalho apresenta um mecanismo de otimização dos recursos de rede através de um algoritmo de determinação da taxa de codificação $(R D A)$ com seleção da taxa baseada na qualidade do sinal recebido. Este mecanismo foi testado em um cenário de emulação de rede IP onde se configuraram diferentes situações de perda de pacotes e atrasos, utilizando-se codificadores de voz multitaxa (G.726 e Speex). O $R D A$ determina a taxa mais adequada baseado no índice $M O S$ obtido pelo $P .563$ e, na ocorrência de atrasos, é acrescentado o $E$ Model. Os resultados obtidos mostram que o $R D A$ proposto tem uma resposta efetiva nos diferentes cenários de rede testados, melhorando a utilização do canal de transmissão.
\end{abstract}

Palavras-Chave-VoIP, RDA, Codec, P.563, E-Model, MOS, socket.

Abstract-This work presents a mechanism for optimization of network resources by using a rate determination algorithm $(R D A)$ with rate selection based on the quality of the received signal. This mechanism was tested in a scenario of IP networking emulation where different situations of packet loss and delay were configured, using multirate speech codecs (G.726 and Speex). The $R D A$ provides a more appropriate rate based on the score obtained by MOS P.563 and, on the occurence of delays, it is supplemented with the E-Model. The results show that the $R D A$ provides effective response in different network scenarios, improving the use of the channel.

Keywords - VoIP, RDA, Codec, P.563, E-Model, MOS, socket.

\section{INTRODUÇÃO}

As comunicações de Voz sobre IP (VoIP) vêm aumentando com o decorrer do tempo e as redes de comunicação estão se tornando cada vez mais congestionadas, ocasionando perda de pacotes e latência crescente nas redes prejudicando diretamente a qualidade das comunicações de voz. O estabelecimento de um mecanismo de controle que otimize a utilização da rede e ao mesmo tempo garanta a melhor qualidade possível do sinal de voz transmitido vem sendo motivo de pesquisa nos últimos anos.

O emprego de codificadores multitaxa nas comunicações de voz possibilita a implementação de algoritmos de determinação da taxa (RDA - Rate Determination Algorithm) que controlam a comutação entre taxas de codificação baseados em diferentes fatores de decisão como as características

Demóstenes Zegarra Rodríguez e Miguel Arjona Ramírez, Laboratório de Processamento de Sinais, Escola Politécnica, Universidade de São Paulo, São Paulo, Brasil, E-mails: demostenes.rodriguez@indt.org.br, miguel@lps.usp.br. Este trabalho recebeu apoio da Fundação de Amparo à Pesquisa do Estado de São Paulo (FAPESP) pelo Proc. 2007/08288-2, do Conselho Nacional de Desenvolvimento Científico e Tecnológico (CNPq) pelo Processo 309249/2008-2 e do Instituto Nokia de Tecnologia. intrínsecas do sinal de voz a ser transmitido ou empregando a informação dos parâmetros de rede. Alguns estudos [1], [2], [3] e [4] são baseados na análise do sinal de voz, como a detecção de atividade de voz $(V A D)$ e algoritmos que determinam o grau de sonoridade de um período do sinal. Isto significa que estes algoritmos $R D A$ determinam a taxa de codificação segundo características do sinal de entrada. Os trabalhos [5], [6] e [7] apresentam um RDA baseado nos parâmetros da rede extraídos das informações que administra o protocolo RTCP [8].

Este trabalho aborda a avaliação do algoritmo de determinação da taxa de codificação, apresentado em [9], em diferentes condições de redes. A taxa de perda de pacotes e os atrasos ponto a ponto são os parâmetros avaliados empregando os codificadores de voz multitaxa ITU-T G.726 [10] e Speex [11].

O fator de decisão deste $R D A$ é a qualidade do sinal medida no ponto da recepção, sendo também possível realizar esta avaliação em qualquer ponto intermediário da transmissão.

Para realização dos testes montou-se um cenário de emulação de rede IP para o estabelecimento de uma chamada VoIP, onde é utilizado um codificador multitaxa. Para avaliação da qualidade do sinal de voz foi empregada a recomendação ITU-T P.563 [12] complementada, no caso de cenários com atraso fixo, pela recomendação ITU-T G.107 [13], sendo transmitido ao emissor o índice $M O S$ utilizando um laço de transmissão, que forma parte da estrutura do mecanismo de controle apresentado neste trabalho. Este laço de transmissão é implementado via socket sobre uma comunicação UDP. Como as ferramentas utilizadas são todas freeware, o cenário de emulação pode ser facilmente montado por demais pesquisadores.

Os resultados mostram uma melhora na utilização do canal de transmissão empregando taxas de codificação menores quando a qualidade do sinal transmitido tem uma pontuação menor, devido a algumas perdas de pacotes ou atrasos na rede, permitindo que um número maior de usuários possa ter acesso à rede. Quando a qualidade do sinal alcança valores acima do intervalo de qualidade definido para uma taxa de codificação o RDA comuta a uma taxa de codificação mais alta garantindo uma melhor qualidade na comunicação VoIP. Ao contrário, quando a qualidade medida está abaixo daquela permitida pela taxa, o RDA comuta a uma taxa mais baixa, liberando capacidade para outros usuários de rede.

Este artigo está dividido em: Seção 2, onde é feito um resumo das ferramentas utilizadas; Seção 3 que apresenta o 
cenário onde são realizados os testes indicando a metodologia seguida e os parâmetros avaliados; Seção 4, onde são apresentados e discutidos os resultados obtidos, e por fim, a Seção 5 que expõe as conclusões e a importância de um mecanismo de controle que otimize a utilização dos recursos da rede.

\section{Elementos do Sistema de Comunicação}

A seguir, serão abordados os principais elementos que fazem parte do sistema de controle implementado neste trabalho: Codificador multitaxa (G.726 e Speex), Avaliadores de qualidade de sinal de voz (P.563 e E-model), RDA e mecanismo de realimentação (socket).

\section{A. Codificador G.726}

O algoritmo original ADPCM (Adaptive Differential PCM) que foi padronizado pela ITU-T em 1984 incluía unicamente a taxa de codificação de $32 \mathrm{kbit} / \mathrm{s}$ e tinha por objetivo codificar a voz na metade da taxa utilizada pelo codificador G.711 [14], mantendo a mesma qualidade do sinal. Este algoritmo está descrito no livro vermelho (Red Book) como a recomendação G.721 [15]. Em 1988 no livro azul (Blue Book) foi publicada a recomendação G.723 [16], que além de incluir a taxa de $32 \mathrm{kbit} / \mathrm{s}$, incluía as taxas de 40 e $24 \mathrm{kbit} / \mathrm{s}$. Em 1990 as Recomendações G.721 e G.723 foram unidas e foi adicionada uma taxa menor igual a $16 \mathrm{kbit} / \mathrm{s}$; esta nova recomendação foi nomeada como G.726 [10]. Estas quatro taxas são geralmente referidas pelo número de bits usados por amostra, que são: 2 bits (16 kbit/s), 3 bits $(24 \mathrm{kbit} / \mathrm{s}), 4$ bits $(32 \mathrm{kbit} / \mathrm{s})$ e 5 bits (40 kbit/s).

\section{B. Codificador Speex}

Speex é um codificador de voz open-source [11]. Este codificador se diferencia dos demais codificadores (exemplo AMR [17]), por não ter sido projetado para comunicações móveis e sim para redes de pacotes e aplicações VoIP suportando diferentes taxas de codificação no intervalo de 2,15 à 24,6 kbit/s. Speex é baseado em um algoritmo CELP (Code-Excited Linear Prediction) que codifica o sinal de voz em quadros de $20 \mathrm{~ms}$, ou seja, 160 amostras a $8 \mathrm{kHz}$, permitindo mudança de taxa a cada $20 \mathrm{~ms}$.

\section{Recomendação ITU-T P.563}

O algoritmo P.563 [12] é aplicável à avaliação da qualidade de voz sem a necessidade de um sinal de referência. Por este motivo, é recomendado para a avaliação não intrusiva da qualidade vocal e para a supervisão de uma rede em funcionamento a partir de um extremo apenas. Esta recomendação não se limita a medições extremo a extremo, podendo ser também utilizada em um ponto qualquer da cadeia de transmissão. A pontuação, assim calculada, é comparável à qualidade percebida por um ouvinte humano que escuta o sinal no ponto de teste. Esta recomendação deve ser utilizada para avaliar a qualidade de fala em aplicações de telefonia com banda de $3,1 \mathrm{kHz}$ (banda estreita).

Deve-se ressaltar que o algoritmo P.563 não proporciona uma avaliação completa da qualidade de transmissão. Assim, só se podem medir os efeitos da distorção unidirecional da voz e do ruído sobre a qualidade vocal, da mesma forma que se analisaria com um teste de audição, que avalia a qualidade na escala da Recomendação ITU-T P.800 [18]. Isto significa que os efeitos de perda de audibilidade, atrasos e qualquer degradação que afete as interações bidirecionais não são observados nas pontuações do P.563.

O sinal de voz digitalizada deve satisfazer os seguintes requisitos:

- Freqüência de amostragem: $8000 \mathrm{~Hz}$;

- Resolução de amplitude PCM linear de 16 bits;

- Duração mínima de 3,0 s e máxima de $20 \mathrm{~s}$.

\section{Recomendação ITU-T G.107}

Esta Recomendação, mais conhecida como E-Model [13], propõe um modelo computacional que mede os efeitos dos parâmetros de uma rede de transporte e do sinal na qualidade de voz transmitida, que é definida pela seguinte equação:

$$
R=R_{o}-I_{s}-I_{d}-I_{e}+A
$$

Onde:

- $R$ : índice de transmissão, tem uma correspondência com a pontuação MOS ITU-T P.800.

- $R_{o}$ : relação sinal-ruído.

- $I_{s}$ : fator de degradações simultâneas. Representa as degradações que afetam simultaneamente o sinal de fala.

- $I_{d}$ : degradação da qualidade devida ao atraso.

- $I_{e}$ : degradação da qualidade por efeito do equipamento (codificador).

- A: fator de melhora.

O valor padrão de $R_{o}$ é 93,2 [13], que é obtido ao se colocar todas as entradas do modelo com seus valores padronizados.

$\mathrm{O}$ parâmetro $I_{s}$ não será considerado nos cálculos deste trabalho, já que este descreve condições que são relacionadas com o sinal, não dependendo da rede de transporte.

O fator $A$, segundo [13], é nulo para redes cabeadas, correspondendo ao cenário de emulação deste trabalho.

$\mathrm{O}$ fator de atraso $I_{d}$ é definido pela composição:

$$
I_{d}=I_{d l e}+I_{d t e}+I_{d d}
$$

As parcelas $I_{d t e}$ e $I_{d l e}$ correspondem aos atrasos devidos aos ecos tanto no emissor quanto no receptor. Estes fatores não serão considerados já que para o cenário de testes se assumirá uma supressão perfeita de eco.

$\mathrm{O}$ fator $I_{d d}$ representa a degradação causada pelos atrasos produzidos na rede, incluso quando se assume uma supressão perfeita de eco, este atraso é expresso por $T_{a}$ (ms) que é configurado segundo o tipo de teste no emulador de rede.

$\mathrm{O} I_{d d}$ é definido a seguir.

Para $T_{a} \leq 100 \mathrm{~ms}: I_{d d}=I_{d}=0$

Para $T_{a}>100 m s$ :

$$
I_{d d}=I_{d}=25\left\{\left(1+d^{6}\right)^{\frac{1}{6}}-3\left(1+\left[\frac{d}{3}\right]^{6}\right)^{\frac{1}{6}}+2\right\}
$$


Com:

$$
d=\frac{\log \left(\frac{T_{a}}{100 m s}\right)}{\log 2}
$$

Com estas considerações, pode-se calcular o parâmetro $R$ em função do valor $R_{o}$, do atraso $\left(I_{d}\right)$ e do fator correspondente ao codificador $\left(I_{e}\right)$.

$$
R=R_{o}-I_{d}-I_{e}
$$

O Fator $R$ está relacionado com o índice $\operatorname{MOS}(M)$ segundo a seguinte equação:

$$
R=3,026 \times M^{3}-25,314 \times M^{2}+87,06 \times M-57,336
$$

\section{E. Algoritmo de Determinação da Taxa}

O algoritmo de determinação da taxa de codificação que opera com a codificadores multitaxa é um mecanismo que depende de fatores externos para poder determinar a taxa de codificação mais adequada em um determinado instante. Estes fatores podem ser características do sinal de entrada como também parâmetros que indicam o estado da rede. A utilização de um RDA em um sistema VoIP tem como objetivo melhorar a qualidade das comunicações e melhorar o uso da rede, podendo adaptar-se a taxa de um codificador multitaxa segundo as condições da rede de uma forma dinâmica e sem a intervenção do usuário de forma que o processo se torna totalmente transparente. Neste trabalho o fator de decisão do $R D A$ é o valor obtido na estimação da qualidade do sinal de voz recebido.

\section{F. Mecanismo de Realimentação}

O laço de transmissão proposto trata-se de uma rotina executada com freqüência dependente do cenário escolhido, podendo ser programada.

Essa rotina tem como função o envio da informação correspondente à qualidade da voz (valor do MOS calculado pelo P.563 e G.113), periodicamente a cada t segundos, desde o receptor até o transmissor. Esta informação é enviada pelo mecanismo de socket [19] através de uma comunicação UDP.

A Figura 1 mostra a rotina (laço) de transmissão implementada neste trabalho; executa-se o P.563 para obter o MOS do lado da máquina cliente que é enviado para a máquina servidora por socket que, ao recebê-lo, comuta para a melhor taxa.

\section{Cenário de Simulação}

A seguir são descritas brevemente as ferramentas utilizadas na implementação do cenário de emulação.

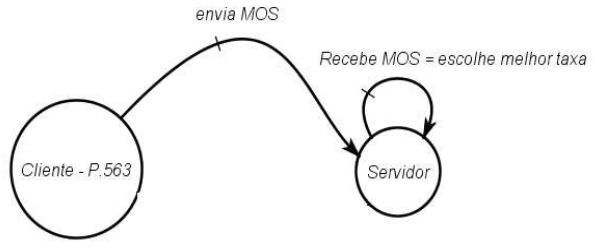

Fig. 1. Diagrama de estados do laço de transmissão.

\section{A. Emulador de Rede NIST Net}

Este emulador [20] de rede foi projetado pelo NIST ( $\mathrm{Na}$ tional Institute of Standards and Technology) para a realização de testes de redes IP, sendo possível emular vários tipos de cenários, como: limitação da taxa de transmissão; atrasos (fixos e variáveis); perdas de pacotes; duplicação de pacotes; aplicação de classe de serviço, etc. Foram implementadas algumas rotinas adicionais para completar algumas funcionalidades que o software nativo (NISTNet) não possuía, como a possibilidade de se mudar a probabilidade de perda de pacotes e atrasos durante uma mesma emulação.

\section{B. Servidor para áudio interativo}

O software utilizado foi o softphone Myphone [21], que realiza comunicações diretas entre dois computadores (PCs) sem servidores intermediários. É um software cliente VoIP de código aberto, desenvolvido pelo grupo OpenH323 da ITU-T. Este softphone permite que o usuário faça a escolha do codificador de voz que será utilizado na comunicação. Adicionaram-se rotinas para a seleção das taxas do codificador empregado.

\section{Analisador de Rede Wireshark}

O Wireshark 0.99.7 System [22] é um software freeware que suporta uma grande quantidade de protocolos, entre eles o IEEE 802.3 [23], empregado nos testes.

\section{Software de gravação de som}

Foi utilizado o software rec do pacote Sox [24], que permite gravar o áudio emitido pela placa de som do computador. $\mathrm{Na}$ realização dos testes, para obter uma qualidade aceitável do arquivo gravado, isolou-se a entrada de áudio, utilizando-se um material isolante na entrada do microfone (porta P2 do computador). Foi implementada uma rotina para poder gravar automaticamente com período $\mathrm{t}$ (para o caso deste trabalho $\mathrm{t}=8$ segundos $)$.

A Figura 2 apresenta o cenário de emulação empregado nos testes realizados.

A seguir é explicado seqüencialmente o procedimento utilizado na realização dos testes, de acordo com a Figura 2.

- Inicialização e programação dos parâmetros de rede no NISNet (para os testes realizados foram incluídos probabilidade de perda de pacotes e atrasos fixos ponto a ponto); 


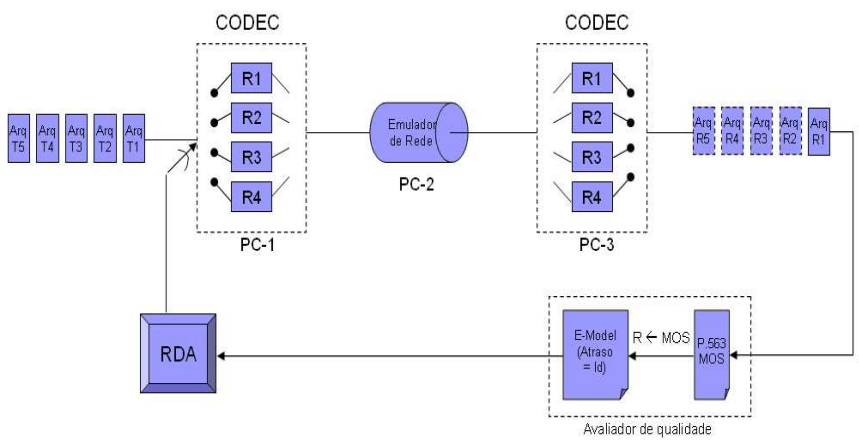

Fig. 2. Cenário de emulação.

- Taxa inicial do codificador $=\mathrm{G} .726=40 \mathrm{kbit} / \mathrm{s}$, Speex $=$ $18,2 \mathrm{kbit} / \mathrm{s}$

- Um arquivo de voz denominado de ArqT-1 é tocado. (Os arquivos utilizados são de 8 segundos e foram extraídos da Recomendação ITU-T P.862 [25]). Este processo é realizado manualmente;

- O sinal codificado é transmitido pela rede (o canal de transmissão emulado) através do softphone empregado;

- Decodificação do sinal transmitido e este foi salvo em um arquivo (ArqR-1), o software de gravação salva arquivos a cada 8 segundos (tempo t que pode ser configurável);

- Estimação da qualidade do sinal de voz recebida utilizando o P.563, resultando um índice MOS. Adicionou-se um código ao P563.c para reduzir a saída dele a um só valor numérico;

- Transmissão deste índice $M O S$ desde o receptor até a fonte utilizando o laço de transmissão;

- Determinação da nova taxa de codificação pelo $R D A$, tendo como fator de decisão o índice $M O S$ recebido;

- Codificação do arquivo seguinte com a nova taxa determinada pelo RDA. Para isso implementou-se um programa com o qual se pode comutar a taxa de codificação no softphone.

\section{REsultados}

Para encontrar os valores limite que determinam os intervalos de $M O S$ correspondentes a cada taxa de codificação no $R D A$, primeiramente, avaliou-se a qualidade do sinal para cada uma das taxas individuais dos dois codificadores multi-taxa empregados nos testes. A Tabela I apresenta os resultados, valores MOS obtidos pela ferramenta P.563 em condições de rede ideais, ou seja, sem atrasos e nem perdas de pacotes.

TABELA I

VALORES MOS COM TAXAS ÚNICAS.

\begin{tabular}{|c|c||c|c|}
\hline \multicolumn{2}{|c|}{ Speex } & \multicolumn{3}{c|}{ G.726 } \\
\hline \hline Taxa (kbit/s) & MOS & Taxa (kbit/s) & MOS \\
\hline 24,6 & 3,95 & 40 & 3,98 \\
\hline 18,2 & 3,91 & 32 & 3,91 \\
\hline 15 & 3,81 & 24 & 3,72 \\
\hline 11 & 3,71 & 16 & 3,19 \\
\hline 8 & 3,45 & & \\
\hline 5,15 & 3,24 & & \\
\hline
\end{tabular}

Tomando como referência os valores $M O S$ da Tabela I, foram construídas tabelas de comutação de taxa dos RDAs para cada um dos codificadores testados, relacionando o valor $\operatorname{MOS}(M)$ com a respectiva taxa $(I)$. Estes algoritmos são:

Para o codificador G.726:

- $M \geq 3,7$ então $I=40 \mathrm{kbit} / \mathrm{s}$;

- $M<3,7$ e $M \geq 3,2$ então $I=32 \mathrm{kbit} / \mathrm{s}$;

- $M<3,2$ e $M \geq 3,0$ então $I=24 \mathrm{kbit} / \mathrm{s}$

- $M<3,0$ então $I=16 \mathrm{kbit} / \mathrm{s}$.

Para o codificador Speex:

- $M \geq 3,8$ então $I=24,6 \mathrm{kbit} / \mathrm{s}$;

- $M<3,8$ e $M \geq 3,6$ então $I=18,2 \mathrm{kbit} / \mathrm{s}$;

- $M<3,6$ e $M \geq 3,4$ então $I=15 \mathrm{kbit} / \mathrm{s}$;

- $M<3,4$ e $M \geq 3,3$ então $I=11 \mathrm{kbit} / \mathrm{s}$;

- $M<3,3$ e $M \geq 3,0$ então $I=8 \mathrm{kbit} / \mathrm{s}$;

- $M<3,0$ então $I=5,15 \mathrm{kbit} / \mathrm{s}$.

Com estes algoritmos implementados no sistema de controle e uma rede sujeita somente à probabilidade de perda de pacotes (PLR), aplicou-se a metodologia anteriormente explicada, obtendo-se os resultados apresentados nas Tabelas II e III.

TABELA II

COMPORTAMENTO DO RDA EM UM CENÁRIO COM PERDA DE PACOTES CODIFICADOR G.726.

\begin{tabular}{|c||c|c|c|c|c|}
\hline & Arq1 & Arq2 & Arq3 & Arq4 & Arq5 \\
\hline \hline Taxa (kbit/s) & 40 & 40 & 32 & 40 & 32 \\
\hline PLR ( $\%)$ & 0 & 8 & 0 & 8 & 0 \\
\hline MOS & 3,98 & 3,28 & 3,86 & 3,21 & 3,87 \\
\hline
\end{tabular}

TABELA III

COMPORTAMENTO DO RDA EM UM CENÁRIO COM PERDA DE PACOTES CODIFICADOR Speex.

\begin{tabular}{|c||c|c|c|c|c|}
\hline & Arq1 & Arq2 & Arq3 & Arq4 & Arq5 \\
\hline Taxa (kbit/s) & 18,2 & 18,2 & 8 & 5,15 & 8 \\
\hline PLR (\%) & 0 & 8 & 6 & 0 & 0 \\
\hline MOS & 3,91 & 3,22 & 2,9 & 3,23 & 3,45 \\
\hline
\end{tabular}

Para determinar como o atraso ponto a ponto afeta a qualidade do sinal transmitido, complementou-se o valor $M O S$ obtido pelo P.563 com as equações do E-Model, seguindo os seguintes passos:

- Transformação do valor MOS num valor $R$ mediante a equação (6), sendo que este valor de $R$ ainda não considera a degradação da qualidade do sinal devida ao atraso.

- Os atrasos, configurados no emulador de rede (NISTNet), são transformados para o fator de degradação devido ao atraso $I_{d}$ mediante as equações (3) e (4).

- Ao valor $R$ encontrado inicialmente se subtrai o fator de degradação devido ao atraso $I_{d}$ encontrado-se $R$ ' que representa a qualidade do sinal recebido considerando as degradações devido aos atrasos de rede.

A Figura 3 apresenta como o atraso ponto a ponto está relacionado com o fator de degradação devido ao atraso $I_{d}$, onde se pode observar que na faixa de 150 a $200 \mathrm{~ms}$ este 
fator começa a incrementar-se afetando a qualidade do sinal recebido, concordando com a recomendação ITU-T G.114 [26].

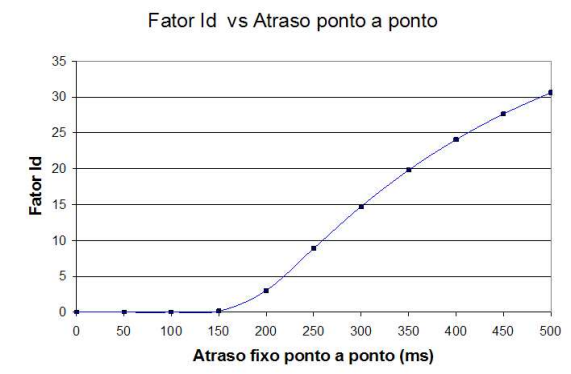

Fig. 3. Relação entre o atraso ponto a ponto e o Fator de Atraso $I_{d}$.

A Figura 4 mostra o comportamento do codificador G.726, nas taxas de 32 e $40 \mathrm{kbit} / \mathrm{s}$ (taxas individuais), em cenários de atraso ponto a ponto e sem considerar perdas de pacotes.

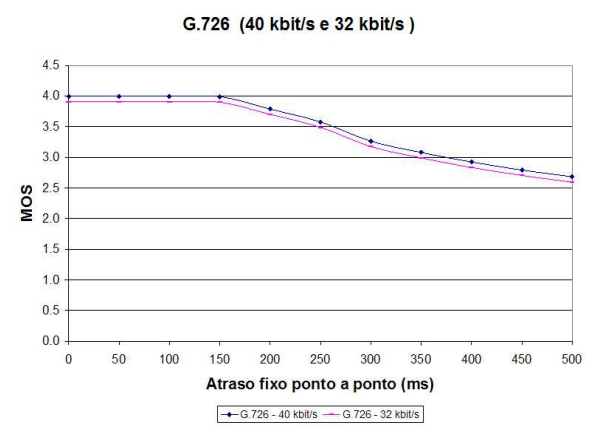

Fig. 4. Comportamento do G.726 em cenários de atrasos fixos ponto a ponto.

Finalmente, para validar a performance do sistema de controle proposto neste trabalho, baseado no RDA, foram testados cenários que incluem atraso e perda de pacotes, tendo como resultados os valores apresentados na Tabela IV. Estes resultados correspondem ao cenário onde se empregou o codificador G.726.

TABELA IV

COMPORTAMENTO DO RDA EM UM CENÁRIO COM PERDA DE PACOTES E ATRASO PARA O CODIFICADOR G.726.

\begin{tabular}{|c||c|c|c|c|c|c|}
\hline & Arq1 & Arq2 & Arq3 & Arq4 & Arq5 & Arq6 \\
\hline \hline Taxa (kbit/s) & 40 & 40 & 32 & 24 & 32 & 40 \\
\hline PLR (\%) & 0 & 8 & 0 & 4 & 0 & 2 \\
\hline Atraso (ms) & 150 & 50 & 300 & 0 & 100 & 200 \\
\hline MOS & 3,93 & 3,33 & 3,15 & 3,42 & 3,88 & 3,41 \\
\hline
\end{tabular}

Com a finalidade de simplificar o processamento no sistema de transmissão (laço de realimentação) os limiares de decisão do $R D A$ foram expressos em função de $R$. Assim para o codificador G.726, o novo RDA seria:

- $R \geq 71,51$ então $I=40 \mathrm{kbit} / \mathrm{s}$;

- $R<71,51$ e $R \geq 61,2$ então $I=32 \mathrm{kbit} / \mathrm{s}$;

- $R<61,2$ e $R \geq 57,72$ então $I=24 \mathrm{kbit} / \mathrm{s}$;

- $R<57,72$ então $I=16 \mathrm{kbit} / \mathrm{s}$.
Estes valores MOS de qualidade podem ser salvos em um arquivo cada vez que a rotina chamar o P.563, dando a possibilidade de gerar relatórios de qualidade que ajudem no gerenciamento da rede com relatório como o da Tabela $\mathrm{V}$ gerado no caso do cenário da Tabela IV.

TABELA V

EXEMPLO DE RELATÓRIO DA QUALIDADE DA REDE.

\begin{tabular}{|c||c|c|}
\hline Data & Hora & MOS \\
\hline \hline $05 / 02 / 2009$ & $17: 45: 08$ & 3,85 \\
\hline $05 / 02 / 2009$ & $17: 45: 16$ & 3,33 \\
\hline $05 / 02 / 2009$ & $17: 45: 24$ & 3,13 \\
\hline $05 / 02 / 2009$ & $17: 45: 32$ & 3,41 \\
\hline $05 / 02 / 2009$ & $17: 45: 40$ & 3,78 \\
\hline $05 / 02 / 2009$ & $17: 45: 48$ & 3,32 \\
\hline
\end{tabular}

As Tabelas VI e VII apresentam resultados obtidos ao comutar as diferentes taxas dos codificadores. Os resultados são razoáveis, pois, quando são empregadas diferentes taxas de codificação, o valor $M O S$ obtido está dentro do intervalo obtido para cada taxa de codificação individual.

TABELA VI

VALORES MOS OBTIDOS PARA CENÁRIOS DE 2 E 3 TAXAS DO CODIFICADORES G.726 (16, 24, 32 E 40 KBIT/s)

\begin{tabular}{|c|c|c||c|c|}
\hline \multicolumn{3}{|c|}{ Rate (kbit/s) } & MOS & Desvio Padrão \\
Taxa1 & Taxa2 & Taxa3 & & \\
\hline \hline 40 & 32 & 24 & 3,909 & 0,0032 \\
\hline 40 & 32 & 16 & 3,362 & 0,0029 \\
\hline 40 & 24 & 16 & 3,315 & 0,0037 \\
\hline 32 & 24 & 16 & 3,299 & 0,0034 \\
\hline 40 & 32 & - & 3,973 & 0,0022 \\
\hline 40 & 24 & - & 3,948 & 0,0024 \\
\hline 40 & 16 & - & 3,926 & 0,0029 \\
\hline 32 & 24 & - & 3,822 & 0,0022 \\
\hline 32 & 16 & - & 3,809 & 0,0027 \\
\hline 24 & 16 & - & 3,497 & 0,0031 \\
\hline
\end{tabular}

TABELA VII

VALORES MOS OBTIDOS PARA CENÁRIOS DE 2 E 3 TAXAS DO CODIFICADORES Speex (5, 8, 15 E 18,2 KBIT/S)

\begin{tabular}{|c|c|c||c|c|}
\hline \multicolumn{2}{|c|}{ Rate (kbit/s) } & MOS & Desvio Padrão \\
Taxa1 & Taxa2 & Taxa3 & & \\
\hline \hline 18,2 & 8 & 5,15 & 3,32 & 0,0039 \\
\hline 18,2 & 8 & - & 3,719 & 0,0021 \\
\hline 18,2 & 5,15 & - & 3,534 & 0,0029 \\
\hline 8 & 5,15 & - & 3,29 & 0,0026 \\
\hline
\end{tabular}

Com a utilização do RDA, pode-se liberar uma determinada quantidade de largura de banda, dependendo das taxas utilizadas (para o caso das taxas testadas com o codificador Speex varia de 3 a 13\%), conseguindo que mais usuários possam utilizar o sistema de comunicação. A qualidade mínima de prestação de serviço pode ser controlada pelo administrador da rede através da configuração do $R D A$. Assim, por exemplo, se o serviço de qualidade que oferece o codificador Speex na taxa $5,15 \mathrm{kbit} / \mathrm{s}$, não segue requisitos mínimos, ela pode ser suprimida. A Tabela VIII apresenta valores de porcentagem 
de largura de banda liberada com o emprego do RDA e o codificador Speex.

\section{TABELA VIII}

OTIMIZAÇÃO DA LARGURA DE BANDA COM O EMPREGO DO RDA.

\begin{tabular}{|c|c|c|c||c|c|}
\hline $\begin{array}{c}\text { Taxa de } \begin{array}{c}\text { Taxificação } \\
\text { (kbit/s) }\end{array} \\
\text { Taxa1 }\end{array}$ & \multicolumn{2}{|c||}{$\begin{array}{c}\text { Tx (kbit/s) } \\
\text { Taxa2 }\end{array}$} & $\begin{array}{c}\text { Taxa de } \\
\text { Tx média } \\
\text { com RDA } \\
\text { (kbit/s) }\end{array}$ & $\begin{array}{c}\text { Largura } \\
\text { de Banda } \\
\text { Liberada } \\
(\%)\end{array}$ \\
\hline \hline 18,20 & 18,20 & 49,40 & 49,40 & 49,40 & 0 \\
\hline 18,20 & 8,00 & 49,40 & 39,20 & 44,30 & 10 \\
\hline 18,20 & 5,15 & 49,40 & 36,35 & 42,88 & 13 \\
\hline 8,00 & 8,00 & 39,20 & 39,20 & 39,20 & 0 \\
\hline 8,00 & 5,15 & 39,20 & 36,35 & 37,78 & 4 \\
\hline 5,15 & 5,15 & 36,35 & 36,35 & 36,35 & 0 \\
\hline
\end{tabular}

A taxa de transmissão [27] é calculada pela equação (7).

$$
T_{t}=\left[\left(T_{p}+C_{i p}\right) / T_{p}\right] \times \mathrm{T}_{c}
$$

Onde:

- $T_{p}=$ Tamanho do pacote (Bytes);

- $T_{c}=$ Taxa de Codificação (bps);

- $T_{t}=$ Taxa de Transmissão (bps);

- $C_{i p}=$ Cabeçalhos RTP/UDP/IP/Camada2 (Bytes).

\section{CONCLUSÕES}

Pode-se concluir que o sistema $R D A$ apresentado neste trabalho otimiza a utilização do canal de transmissão, escolhendo para um cenário de rede a taxa de codificação mais adequada determinada com base na qualidade do sinal no ponto de recepção. Desta forma pode-se evitar uma sobrecarga na rede e garantir a mais alta qualidade de comunicação que as condições de rede permitam.

Ao melhorar a utilização da rede, garante-se que um número maior de usuários possa realizar comunicações VoIP, sendo mais significativo quando se tratar de um número alto de usuários.

O mecanismo de $R D A$ apresentado é totalmente automático e autônomo, ou seja, o processo de controle não é perceptível ao usuário.

O Sistema de Controle permite monitorar a qualidade da rede já que é possível gerar relatórios de qualidade do sinal para um determinado instante.

Cabe ressaltar que os resultados obtidos nestes testes são compatíveis, pois, quando são empregadas diferentes taxas de codificação, o valor $M O S$ obtido está dentro do intervalo de valores MOS obtidos para cada taxa de codificação individual.

\section{AgRADECIMENTOS}

Os autores agradecem o Laboratório de Processamento de Sinais da Universidade de São Paulo e o Laboratório de Telecomunicações do Instituto Nokia de Tecnologia pela motivação à pesquisa na área de Sistemas de Telecomunicações.

\section{REFERÊNCIAS}

[1] Paksoy, E.; Gersho, A., Multimode and variable-rate coding of speech, in Speech Coding and Synthesis. Eds. W. B. Kleijn and K. K. Paliwal, Elsevier, pp. 257-288, 1995.

[2] Gersho, A.; Paksoy, E., Variable rate speech coding for cellular networks, in Speech and Audio Coding for Wireless and Network Applications. Eds. B. Atal, V. Cuperman, Kluwer Academic Publishers, pp. 77-84, 1993.

[3] Paulus, J. W., Variable bitrate wideband speech coding using perceptually motivated thresholds. IEEE Workshop on Speech Coding for Telecommunications, pp. 3536, Annapolis, Maryland, USA, September 1995.

[4] Hermansky, H., Perceptual linear predictive (PLP) analysis of speech. Journal of the Acoustical Society of America, vol. 87, no. 4, pp. 17381752, April 1990.

[5] Komisarczuk, P.; Koudrin, A., Effect of rerouting on NGN VoIP Quality. IEEE, Australian Telecomunication Networks and Aplications Conference, pp.364-370, December 2007.

[6] Anna Sfairopoulou, Carlos Macian and Boris Bellalta. QoS Adaptation in SIP-based VoIP calls in Multi-rate IEEE 802.11 Enviroments. IEEE Wireless Communications Systems, vol. 4606, Valencia, Spain, September 2006 .

[7] P. Galiotos, T. D. Agiuklas, D. Arkadianos, QoS Management for an enhanced VoIP platform using R-factor and Network Load Estimation functionality. IEEE - High Speed Networks and Multimedia Communications 5th IEEE International Conference, pp. 305 314, Athens Greece, November 2002.

[8] IETF Rec. 3550, A Transport Protocol for Real-Time Applications, Jul. 2003, www.ietf.org/rfc/rfc3550.txt.

[9] Zegarra Rodríguez, D. ; Arjona Ramírez, M. , VoIP Quality Improvement with a Rate-determination Algorithm. International Workshop on Telecommunications, São Paulo - Brazil, September 2009, v. 1. p. 30-34.

[10] ITU-T Rec. G.726, General Aspects of Digital Transmission Systems, Terminal Equipment - 40, 32, 24,16 kbit/s Adaptive Differential Pulse Code Modulation (ADPCM), 1990, http://www.itu.int/rec/T-RECG.726/en.

[11] Jean Valin, The speex codec manual, http://www.speex.org/docs/.

[12] ITU-T Rec. P.563, Single-ended method for objective speech quality assessment in narrow-band telephony applications, May. 2004. Disponível em: www.itu.int/rec/T-REC-P.563/en.

[13] ITU-T Rec. G.107, The E-model, a computational model for use in transmission planning. Mar. 2005. http://www.itu.int/rec/T-REC-G.107/en.

[14] ITU-T Rec. G.711, General Aspects of Digital Transmission Systems Terminal Equipments - Pulse Code Modulation (PCM) of Voice Frequencies. 1972. http://www.itu.int/rec/T-REC-G.711/en.

[15] CCITT R. G.721, 32kbit/s adaptive differential pulse code modulation (ADPCM). IXth Plenary Assembly, Melbourne, 14-25 Nov. 1988, Blue Book.

[16] ITU-T Rec. G.723.1, General Aspects of Digital Transmission Systems - Dual Rate Speech Coder for Multimedia Communications Transmitting at 5.3 and $6.3 \mathrm{kbit} / \mathrm{s}$. May. 2006. http://www.itu.int/rec/T-RECG.723.1/en.

[17] 3GPP Rec. TS 26.071 Mandatory speech CODEC speech processing functions; AMR speech Codec; General description. Disponível em http://www.3gpp.org/ftp/Specs/html-info/26-series.htm

[18] ITU-T Rec. P.800, Methods for subjective determination of transmission quality. Aug. 1996. Disponível em: www.itu.int/rec/T-REC-P.800/en.

[19] Homayunfar, K., Rate adaptative speech coding for Universal Multimedia Access. IEEE Signal Processing Magazine, pp. 30-39, vol. 20, no. 2 , March 2003

[20] NISTNET, http://www x.antd.nist.gov/nistnet/.

[21] Softphone Myphone, http://myphone.sourceforge.net/.

[22] Wireshark, http://www.wireshark.org/download.html.

[23] IEEE Ethernet Working Group, CSMA/CD Access Method, Diponível em: http://standards.ieee.org/getieee802/802.3.html

[24] Sox - Sound eXchange : universal sound sample translator. linux.die.net/man/1/rec

[25] ITU-T Rec. P.862, Perceptual evaluation of speech quality (PESQ): An objective method for end-to-end speech quality assessment of narrowband telephone networks and speech codecs. http://www.itu.int/rec/TREC-P.862/en.

[26] ITU-T Rec. G.114 , One way transmission time. May. 2003. http://www.itu.int/rec/T-REC-G.114/en.

[27] Wendell Odom, CCENT/CCNA ICND2, Official Exam Certification Guide. Second Edition. Cisco Systems, Sep. 2007. 